\title{
The most evolved sources in the Hi-GAL survey
}

\author{
Elia D.* \\ INAF-IAPS, Roma, Italy
}

\begin{abstract}
Far-infrared and submillimetre surveys as the Herschel Galactic Plane Infrared Survey (HiGAL) represent an irreplaceable knowledge base about early phases of star formation, permitting statistical analysis based on thousands of Galaxy-wide distributed sources. Those with a regular spectral energy distribution in the Herschel wavelength range 70-500 $\mu \mathrm{m}$ span a variety of evolutionary stages, from quiescent to star forming clumps and, within the latter class, from mid-infrared dark clumps to sources appearing very bright also at shorter wavelengths (e.g. Spitzer $24 \mu \mathrm{m}$ ). A fraction of these clumps hosts the formation of high mass stars, which are expected to reach the zero age main sequence and to develop a HII region in their surroundings while they are still embedded in their parental large-scale dusty envelope. This paper aims at selecting and studying in detail a robust sample of Hi-GAL clumps supposed to be candidate to host a HII region in their interior. They are expected to be the most evolved sources in the Hi-GAL catalogue. The Galactic locations and the physical properties (temperature, mass, bolometric luminosity and temperature, and surface density) of these sources are discussed here. The large number (1199) of selected sources constitutes an important starting point for planning further interferometric programs, aimed at resolving possible cores hosting a young high-mass star.
\end{abstract}

Keywords: Stars: formation - ISM: clouds - ISM: dust - Galaxy: local interstellar matter - Infrared: $I S M$

\section{Introduction}

The influence of massive stars $\left(M>8 \mathrm{M}_{\odot}\right)$ extends well beyond their immediate neighbourhoods, having effects on the interstellar medium and Galactic evolution. Through strong stellar winds and, finally, supernovae explosions, they enrich the interstellar medium by injecting mechanical energy and supplying it with processed material. Furthermore, their radiation output is the responsible of the ionization of the surrounding gas. Therefore they influence the subsequent star formation in their environments, being even a possible triggering mechanism of it. On a larger scale, stellar winds from massive stars and and supernovae lead to the formation of super-bubbles.

Despite their importance, massive stars represent a minority of the Galactic population, as it can be deduced from the slope of the well-known stellar initial mass function (Kroupa, 2001, Salpeter, 1955). Their evolutionary time scales, including that of the formation process, are considerably shorter than in their low-mass counterparts, so that the details of the phases of massive star formation are still elusive. Consequently, the need of observing sites of massive star formation to probe the early phases of this process and their connection with the surrounding environment clashes with the low availability of such regions in the Solar neighbourhoods: the large majority of known massive star forming regions are located beyond a few kiloparsecs from the Sun, and prospectively distributed on the Galactic plane.

In this respect, unbiased global-scale surveys of the Milky Way Galactic plane from infrared to radio wavelengths represent, compared with studies dedicated to single regions, a breakthrough in the direction of studying the impact of massive star formation on the whole Galactic ecosystem, and of offering the possibility of bridging the gap between local and extragalactic star formation studies.

*davide.elia@inaf.it 
The most evolved sources in the Hi-GAL survey

The WISE, Spitzer, and Herschel mid- to far-infrared continuum surveys, together with surveys from ground-based facilities at millimetre and radio wavelengths, enabled us to reveal the Galactic distribution of dust, and to measure its physical properties on all scales of the ISM, from diffuse clouds to hundreds of thousands of dense clumps. Spectroscopic surveys in various atomic and molecular tracers provide, in turn, the chemical characteristics of dense clumps, as well as kinematic information which is essential to derive heliocentric distances and other distance-dependent parameters.

The Herschel Infrared Galactic Plane Survey (Hi-GAL, Molinari et al., 2010) plays a major role in this scenario, thanks to the unprecedented quality of Herschel observations, in terms of resolution, sensitivity and dynamical range, in a crucial spectral range (five photometric bands between 70 and $500 \mu \mathrm{m}$ ) for studying emission from cold dust in interstellar clouds. At these wavelengths, the early stages of star formation can be observed in almost point-like condensations, hereafter compact sources, that can correspond to cores or clumps, depending on their heliocentric distance (e.g., Elia et al., 2013, 2017, hereafter El17). A quiescent vs active character with regard to star formation can be attributed to such sources based on spectroscopic signposts (e.g., Giannetti et al., 2013), but also on a selfconsistent photometric criterion that could be rougher but certainly applicable to all sources when spectroscopy is not available: a detection at $70 \mu \mathrm{m}$ indicates a proto-stellar character, otherwise the source is classified as starless (Dunham et al., 2008, Elia et al., 2013, Giannini et al., 2012, Könyves et al., 2015).

A complete catalogue of Hi-GAL clump physical properties at inner longitudes $\left(-71^{\circ} \leq \ell \leq+68^{\circ}\right)$ was released by El17, who identified a set of distance-independent parameters derived from spectral energy distributions (SED), namely the modified black body temperature $T$, the ratio of the bolometric luminosity to the mass $L / M$, the ratio of the bolometric luminosity to the portion in the sub-millimetre $L / L_{\mathrm{smm}}$, and the bolometric temperature $T_{\mathrm{bol}}$, able to provide a first evolutionary classification. In light of these parameters, a good degree of segregation was found between the starless and the protostellar classes. In particular, distributions of these quantities for proto-stellar sources appear generally broader than for starless sources, testifying a certain diversification of evolutionary stages within the phase globally labelled as proto-stellar.

This is consistent with other evidences offered by the comparison with other tracers: Molinari et al. (2016) calibrated the $L / M$ ratio through $\mathrm{CH} 3 \mathrm{C} 2 \mathrm{H}(12-11)$ line observations. They find that this line is detected in sources with $L / M>1 \mathrm{~L}_{\odot} / \mathrm{M}_{\odot}$ (corresponding to a temperature $T<30 \mathrm{~K}$ in the inner part of the clump), and the temperature indicated by this tracer starts to increase at $L / M>10 \mathrm{~L}_{\odot} / \mathrm{M}_{\odot}$, so that this threshold is interpreted as the first appearance of one or more zero-age main sequence stars in the clump.

Cesaroni et al. (2015), analysing 200 Hi-GAL counterparts of CORNISH survey (carried out with VLA at $5 \mathrm{GHz}$ and covering the area $10^{\circ}<\ell<65^{\circ},|b|<1^{\circ}$, Hoare et al., 2012, Purcell et al., 2013) sources classified as "ultracompact" and "compact" HiI regions, found a $L / M$ ratio ranging from 1 to $250 \mathrm{~L}_{\odot} / \mathrm{M}_{\odot}$.

The work of Cesaroni et al. (2015) inspired El17 to establish a criterion to identify HiI region candidates also in areas of the Galactic plane not covered by CORNISH and other radio surveys in general, and to discuss the statistics of their physical parameters in comparison with those of earlier evolutionary classes. However, locations of these sources were not shown, and internal correlations among physical parameters were not developed in detail. The aim of this paper is therefore to better describe and characterize the sample of Hi-GAL HII region candidates, presenting original analysis of their physical parameters estimated from infrared photometry.

The paper is organised as follows: in Sect. 2 the sample selection process is illustrated, and the Galactic locations and heliocentric distances of selected sources are shown; in Sect. 3 a discussion of different source physical parameters is provided; finally, in Sect. 4 main conclusions are summarised.

\section{Sample selection}

In El17, candidate HiI regions have been identified starting from the work of Cesaroni et al. (2015), who built the histogram of the logarithm of the Herschel-based $L / M$ ratio for these sources, where $M$ is derived from the modified black body fit of the SED at $\lambda \geq 160 \mu \mathrm{m}$, and the luminosity from 
The most evolved sources in the Hi-GAL survey

the integral of the entire observed $\mathrm{SED}^{1}$. The ascending part of the distribution, corresponding to low values of $L / M$, can be interpreted as a genuine increase in cases at increasing $L / M$. On the contrary, the descending part to the right of the peak is due to a completeness effect: more evolved sources, at large $L / M$ might not be included in the Hi-GAL catalogue of El17. Indeed, among the requirements for sources to be listed in this catalogue is the availability of a detection at 250 and $350 \mu \mathrm{m}$, which is necessary to ensure a reliable fit to the SED, but favours the colder sources and rejects flux combinations such as 70 $\mu$ m-only, or 70-160 $\mu$ m-only, or 70-160-250 $\mu$ m-only, which correspond to warmer and more evolved sources.

El17 therefore pursue the idea of using the peak of this distribution as a conservative minimum threshold to identify candidate Hir regions in their catalogue even at longitudes not covered by CORNISH. To identify this threshold more precisely, they plotted again the histogram of this distribution in bins of 0.1 and found a peak at 1.35 , which corresponds to $22.4 \mathrm{~L}_{\odot} / \mathrm{M}_{\odot}$ (note that this value is also compatible with the aforementioned threshold of $L / M>10 \mathrm{~L}_{\odot} / \mathrm{M}_{\odot}$ by Molinari et al., 2016). Applying this threshold to the sub-sample of proto-stellar clumps of their catalogue, El17 labelled 2377 sources as candidate HiI regions (Fig. 1).

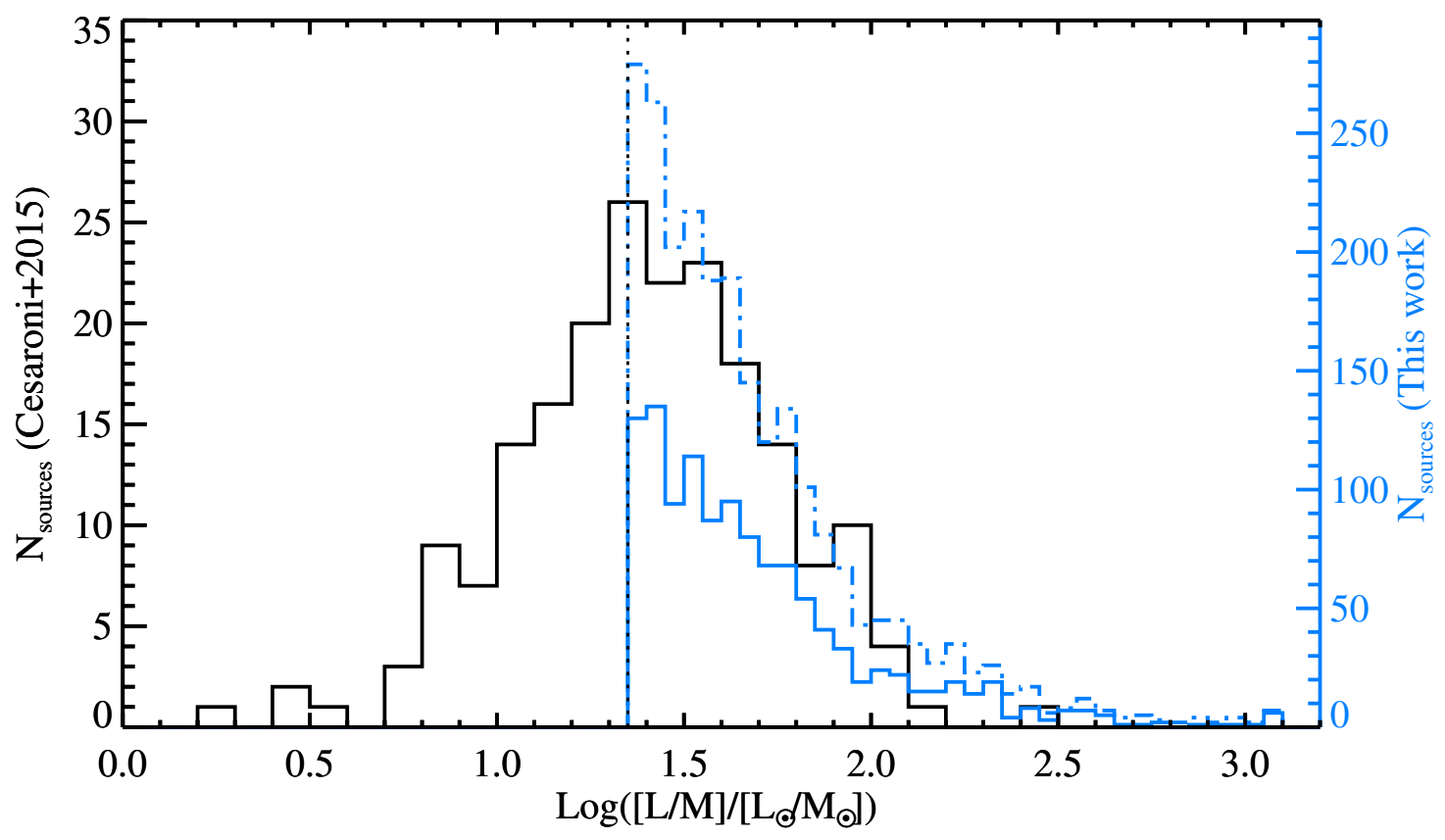

Figure 1. Black solid histogram: distribution, in logarithmic bins of 0.1 , of the $L / M$ quantity for Hi-GAL counterparts of CORNISH sources (data from Cesaroni et al., 2015). The peak position is marked with a vertical dashed line. Cyan dotted-dashed histogram: distribution, in logarithmic bins of 0.05 , of the same parameter, but for all candidate Hil regions from the catalogue of El17, i.e. sources with $L / M>22.4 \mathrm{~L}_{\odot} / \mathrm{M}_{\odot}$. This histogram refers to the scale on the right $y$-axis. Cyan color is used here and throughout this paper for indicating HII region candidates for the sake of uniformity with notation of El17. Cyan solid histogram: same as dotted-dashed histogram, but only for sources selected for the present analysis, namely those fulfilling the condition $L>1448 \mathrm{~L}_{\odot}$.

In this work, a further selection is carried out, adding a further conservative constraint on the global luminosity, which must be compatible with the presence of a central high-mass star, namely $L>8^{3.5}=1448 \mathrm{~L}_{\odot}$. The distribution of $L / M$ for the selected sample of sources is also shown in Fig. 1. It can be noted that the second step of selection based on the luminosity filtered out about the $50 \%$ of sources, at least in the most populated and statistically relevant bins. A commented example

\footnotetext{
${ }^{1} \mathrm{Hi}-$ GAL SEDs were complemented in the mid-infrared, where possible, with fluxes at 21, 22, and $24 \mu \mathrm{m}$ from $M S X$ (Egan et al., 2003), WISE (Wright et al., 2010), and Spitzer-MIPSGAL (Gutermuth \& Heyer, 2015) surveys, respectively. Mid-infared fluxes are generally involved in the calculation of evolutionary parameters and produce an enhancement of them with respect to pure Hi-GAL SEDs; only modified black body temperature estimation is independent from midinfrared fluxes.
} 
The most evolved sources in the Hi-GAL survey

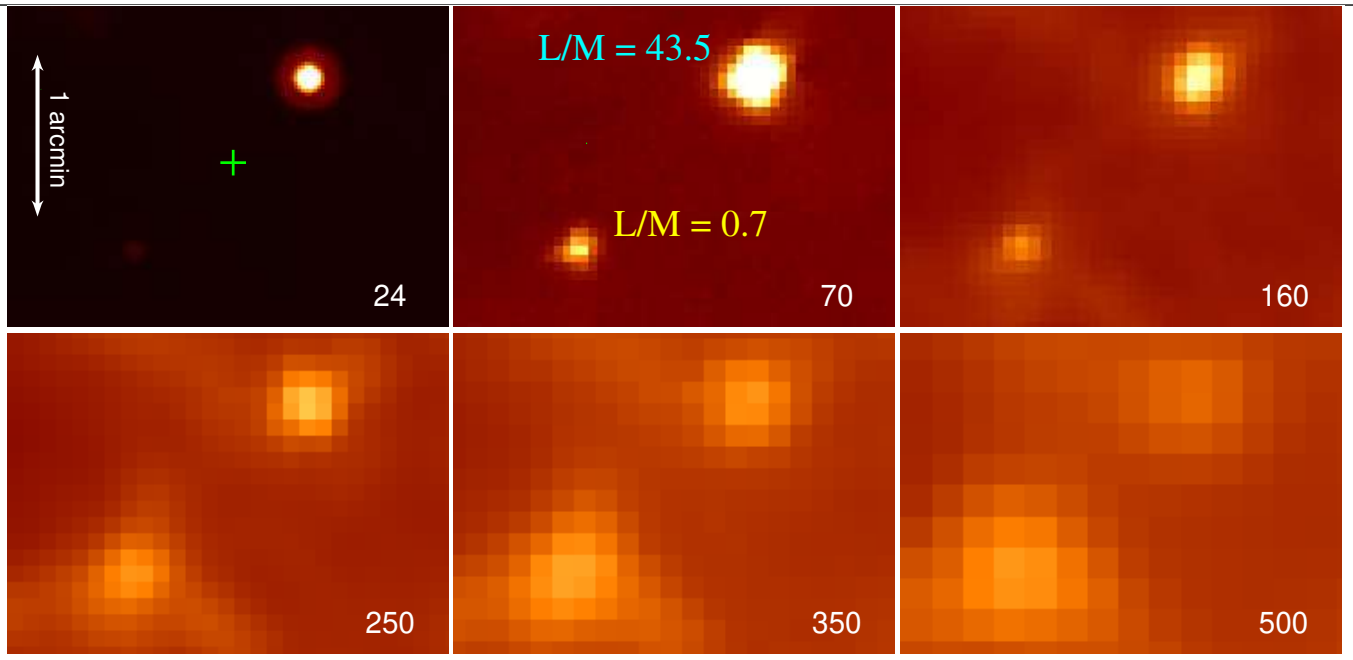

Figure 2. Multi-wavelength view of a Hi-GAL source selected as a Hit region candidate. From top-left to bottom-right frame: MIPSGAL $24 \mu \mathrm{m}$ image and Hi-GAL 70, 160, 250, 350, and $500 \mu \mathrm{m}$ images, respectively. The wavelength is specified in the bottom-right corner of each frame. In the $24 \mu \mathrm{m}$ frame the center of the image is indicated with a green cross and corresponds to coordinates $\ell=17.20468^{\circ}$ and $b=+0.11299^{\circ}$. The angular scale of 1 arcmin is represented, in the same frame, with a white vertical segment. The selected source is located towards the top-right corner, and indicated with its peculiar $L / M=43.5 \mathrm{~L}_{\odot} / \mathrm{M}_{\odot}$ in the $70 \mu \mathrm{m}$ frame. Another protostellar source, ruled out by the selection procedure, is located towards the bottom-left corner, and indicated with $L / M=0.7 \mathrm{~L}_{\odot} / \mathrm{M}_{\odot}$. It can be seen that the source with high $L / M$ is bright at $24 \mu \mathrm{m}$, while the source at low $L / M$ is dark, starting to be visible at $70 \mu \mathrm{m}$. Conversely, at the two longest wavelengths, 350 and $500 \mu \mathrm{m}$, the low- $L / M$ source appears more extended and globally brighter than the high- $L / M$ one.

of selected source is presented in Fig. 2, as it appears at wavelengths from 24 to $500 \mu \mathrm{m}$.

In El17 a comparison between Herschel colors of HiI region candidates and of Hi-GAL counterparts of 16 bona-fide Hiı regions studied by Paladini et al. (2012) was carried out. The majority of candidates populate the region of the $\log \left(F_{\nu, 70} / F_{\nu, 160}\right)$ vs $\log \left(F_{\nu, 250} / F_{\nu, 500}\right)$ diagram occupied by the sources of Paladini et al. (2012), delimited by $\log \left(F_{\nu, 70} / F_{\nu, 160}\right)>-0.45$ and $\log \left(F_{\nu, 250} / F_{\nu, 500}\right)>0.6$. In the sample considered in this paper, 1115 sources (i.e. $93 \%$ of the total) fulfil both these conditions.

The condition on the luminosity implicitly imposes that selected sources have a heliocentric distance estimate. In El17 only 57065 out of 100922 catalogue sources are provided with a distance, and, in particular kinematic distances in the longitude range $-10 .^{\circ} 2<\ell<14 .^{\circ} 0$ are not calculated, due to the difficulty in estimating them for sources in the direction of the Galactic Centre. The resulting distribution of sources in the Galactic plane is shown in Fig. 3. As already noted by El17 for the entire Hi-GAL source sample, the disposition of HiI region candidates does not show a clear separation between populated spiral arm locations against void inter-arm regions. However, in limited portions of the displayed arms (from Hou et al., 2009) overdensities of Hil region candidates are recognizable, both in the first and in the fourth quadrant. No specific trends are seen between source locations and their $L / M$.

Finally, in Fig. 4 the mass vs heliocentric distance plot for both the selected and ruled out sources is shown. The cut imposed on luminosity filtered out essentially sources at short distances and/or low masses, so that the $95 \%$ of selected sources lie at $M>20 \mathrm{M}_{\odot}$ and $d>3 \mathrm{kpc}$, respectively, compared to $8 \%$ for rejected sources.

\section{Hi-GAL candidate HII regions physical parameters}

The average dust temperature estimated through the modified black body fit has been proven by El17 to be an evolutionary indicator, although, compared with other parameters, it shows a lower power of separation between different stages. This is essentially due to the fact that the component 


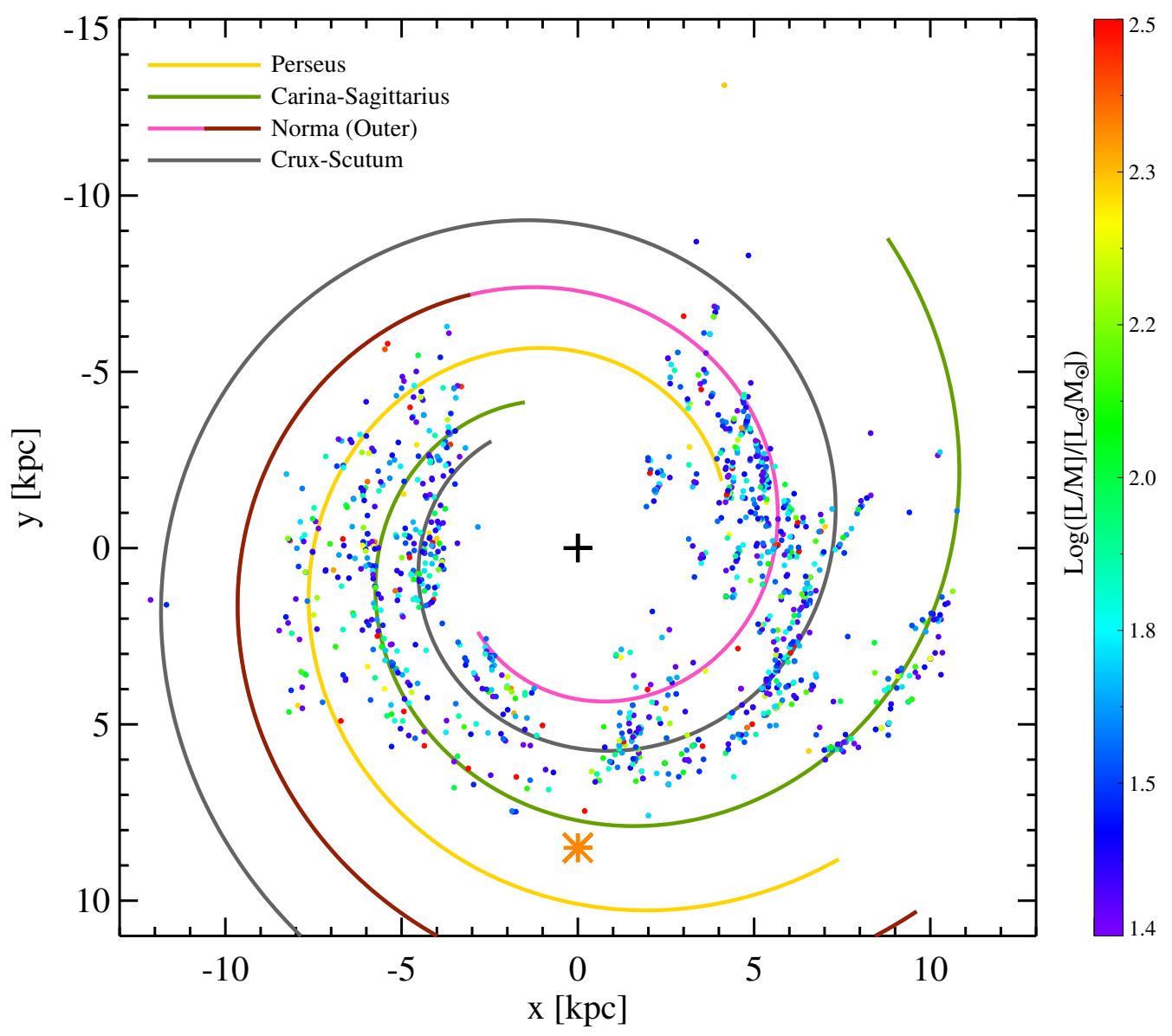

Figure 3. Plot of the positions in the Galactic plane of the Hi-GAL Hil region candidates selected in this work. Symbol colors are encoded based on the logarithm of $L / M$, as reported in the color bar on the right. The inner zone $\left(-10 .^{\circ} 2<\ell<14 .^{\circ} 0\right)$ is devoid of points, because distances were not reported in El17. The Galactic Centre is indicated with a large plus symbol at coordinates $[x, y]=[0,0]$, and the Sun with an orange asterisk at coordinates [0,8.5]. Spiral arms, from the four-arm Milky Way prescription of Hou et al. (2009), are plotted with different colours, according to the legend in the upper left corner. In particular, the Norma arm is represented using two colours: magenta for the inner part of the arm and brown for the portion of it generally designated as the Outer arm (see Momany et al., 2006). There is no link between the arm color choice and the color coding for source symbols.

observed at $\lambda \geq 160 \mu \mathrm{m}$ corresponds to the outer and coldest layer of a clump, which is only marginally influenced by the central source, if any.

Median kinetic temperatures of $23.3 \mathrm{~K}$ and $24 \mathrm{~K}$ for a set of 16 and 170 clumps known to host a HII region in their interior were estimated by Hofner et al. (2000) and Urquhart et al. (2013), respectively. These values are similar to the median temperature of $24.4 \mathrm{~K}$ found for the sources considered in this work, whose temperature distribution is shown in Fig. 5, left panel.

Another evolutionary parameter is the bolometric temperature, defined by Myers \& Ladd (1993) as

$$
T_{\text {bol }}=1.25 \times 10^{-11} \times \frac{\int_{0}^{\infty} \nu F_{\nu}}{\int_{0}^{\infty} F_{\nu}}
$$

(where $T_{\text {bol }}$ is expressed in $\mathrm{K}$ and $\nu$ in $\mathrm{Hz}$ ), as a way to quantify spectral redness as the temperature of a blackbody whose spectrum has the same mean frequency (weighted with fluxes) of an observed SED. For a single star-forming core, and in the low-mass regime, Chen et al. (1995) identified a threshold of $T_{\text {bol }}=70 \mathrm{~K}$ for separating Class 0 from Class I young stellar objects, according to the classification introduced by Lada \& Wilking (1984), Lada (1987), and André et al. (1993).

For entire clumps, however, what is expected is that contained, unresolved cores can have different Elia D. 


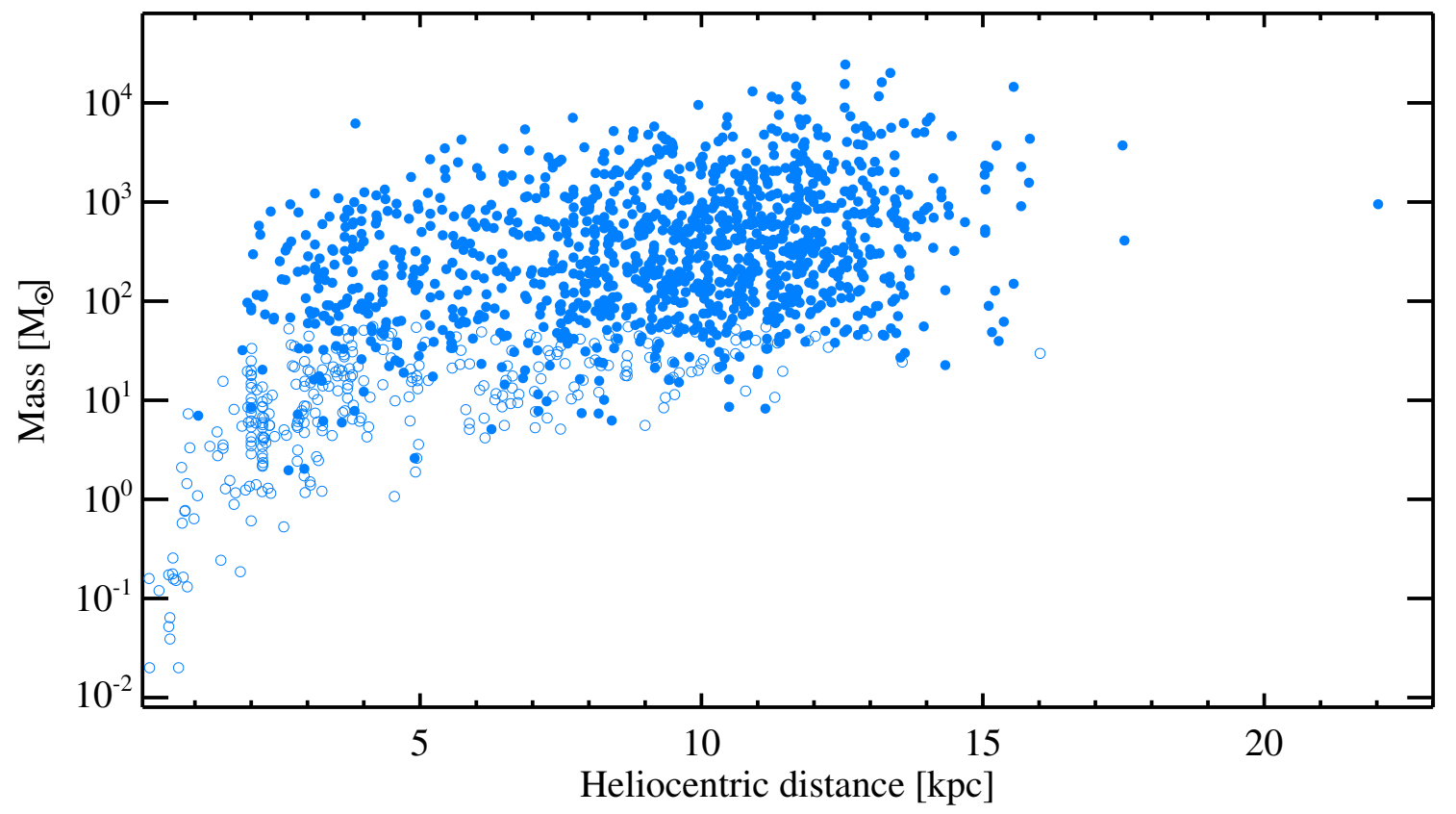

Figure 4. Mass vs heliocentric distance for Hi-GAL HiI region candidate sources selected for the analysis presented in this work (filled circles) and filtered out (open circles).
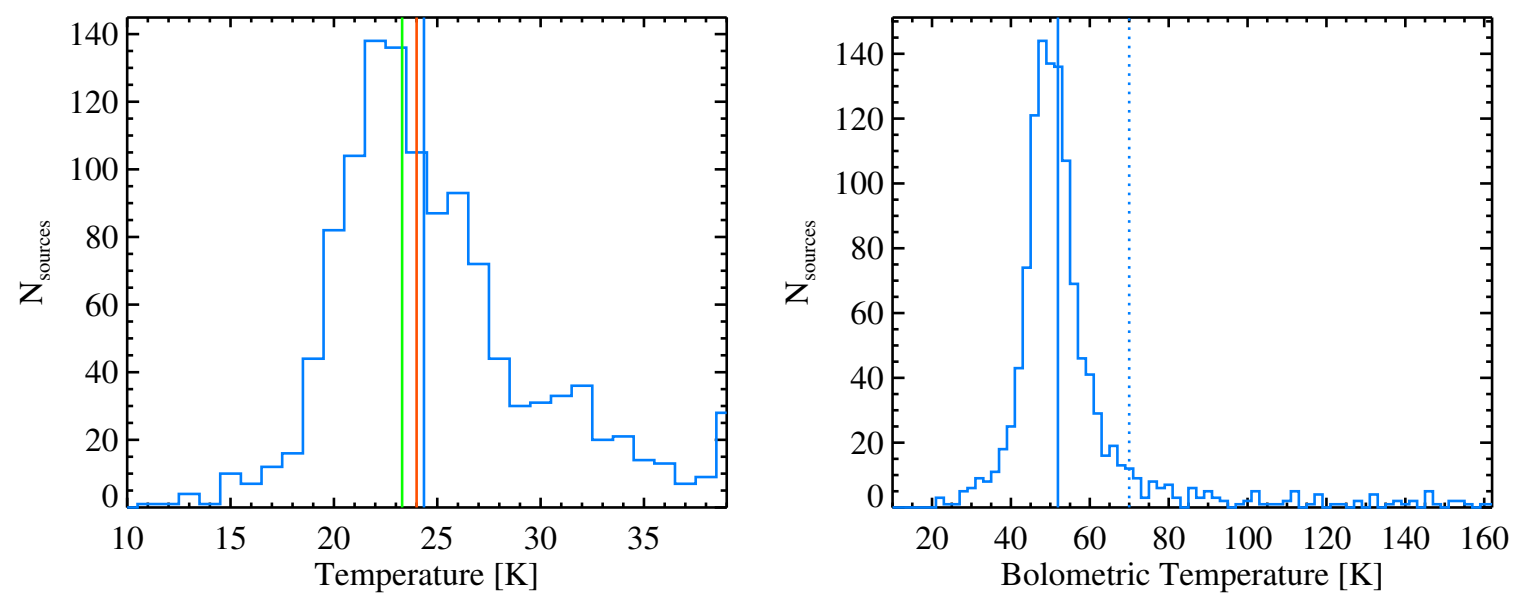

Figure 5. Left: histogram of modified black body fit temperature, in bins of $1 \mathrm{~K}$, for Hi-GAL HII region candidates considered in this work. The cyan vertical line represents the median of this distribution, whereas the green and red vertical lines represent the median kinetic temperatures for the clump samples of Urquhart et al. (2013) and Hofner et al. (2000), respectively. Right: same as left panel, but for bolometric temperature, in bins of $2 \mathrm{~K}$. The cyan solid and dotted vertical lines represent the median of this distribution and the $T_{\mathrm{bol}}=70 \mathrm{~K}$ threshold of Chen et al. (1995) for Class I sources, respectively.

evolutionary stages and this, together with the presence of quiescent inter-core material, leads to underestimate global evolutionary indicators, including $T_{\text {bol }}$. For the selected sample of Hi-GAL HiI region candidates, the median bolometric temperature id $52 \mathrm{~K}$, and about $10 \%$ of sources have $T_{\text {bol }}=70 \mathrm{~K}$ (Fig. 5, right panel), which corresponds to affirm that at least $10 \%$ of these clumps contain at least a young stellar object compatible with a classification of Class I (or equivalent in the high-mass regime).

Fig. 6 contains the plot of $T_{\text {bol }}$ vs $L / M$ for Hil region candidates. This diagram was not explicitly shown by El17, who plotted this relation for the overall class of proto-stellar clumps. First, the different dynamical range for these two observables is evident, with $L / M$ and $T_{\text {bol }}$ spanning about two orders of magnitude and only half order of magnitude, respectively. This implies that any possible variation 


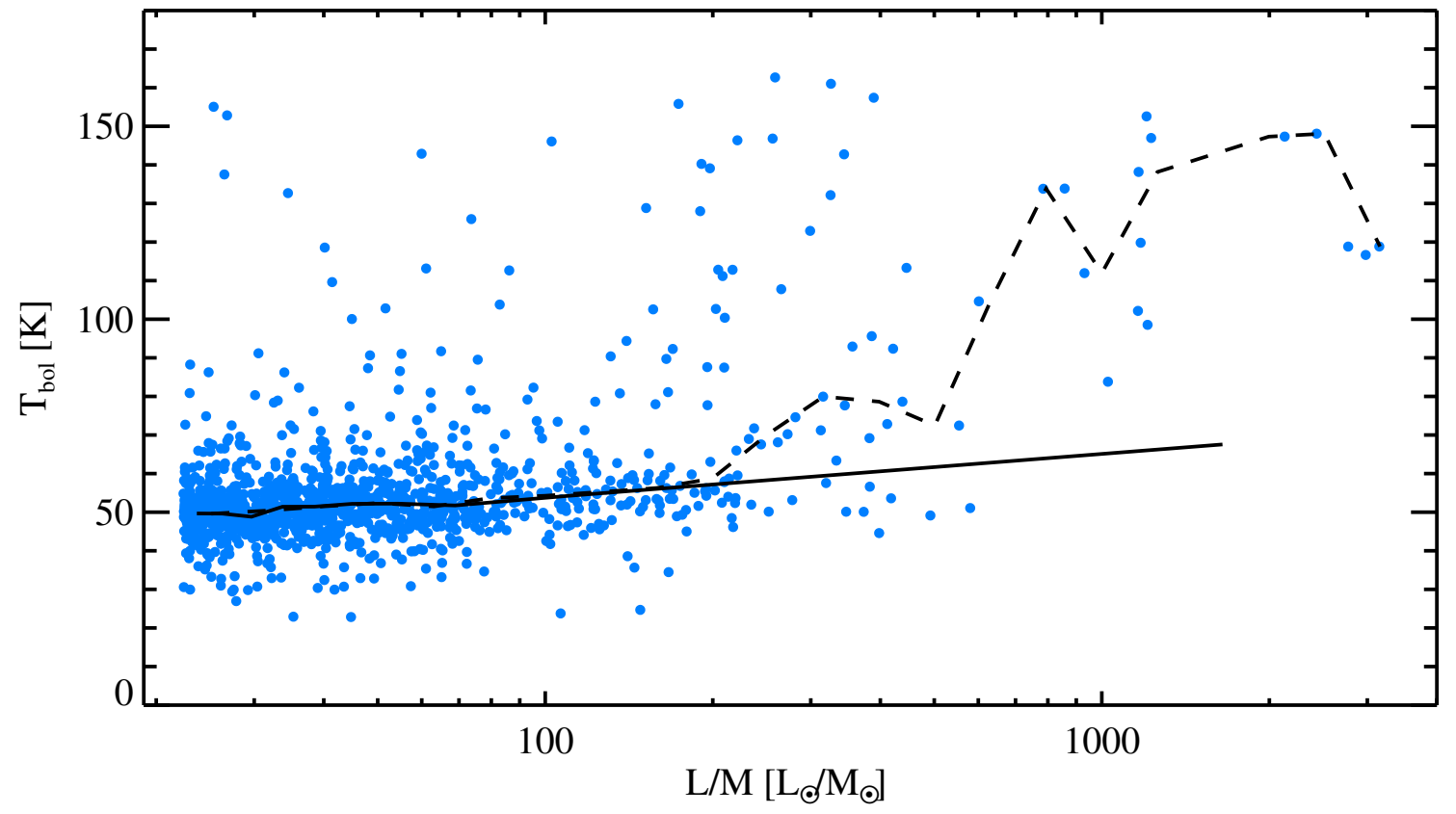

Figure 6. Bolometric temperature vs ratio of bolometric luminosity to mass for Hi-GAL HII region candidate sources selected for the analysis presented in this work. The two black lines connect the medians of $T_{\mathrm{bol}}$ in bins of $\log (L / M)$ with fixed width 0.1 (dashed), and in bins delimited by percentiles from $0 \%$ to $100 \%$ in steps of $10 \%$ (solid), respectively.

of $T_{\text {bol }}$ as a function of $L / M$ is expected to be very shallow. Even, Williams et al. (2004) did not recognize, in a similar plot for 19 objects, a clear trend between these two quantities. On the contrary, Fig. 6 shows a slightly increasing behaviour of $T_{\text {bol }}$ for $L / M<600 \mathrm{~L}_{\odot} / \mathrm{M}_{\odot}$, net of outliers found at high bolometric temperature (say $T_{\text {bol }}>70 \mathrm{~K}$ ). Moreover, for $L / M>600 \mathrm{~L}_{\odot} / \mathrm{M}_{\odot}$, only values of $T_{\mathrm{bol}}>80 \mathrm{~K}$ are found. To better highlight a possible global trend, medians of $T_{\mathrm{bol}}$ in 0.1-wide logarithmic bins of $L / M$ are connected with a dashed line in Fig. 6, so that the two aforementioned regimes can be easily noticed. However, to give the same statistical relevance to all points, the medians of $T_{\text {bol }}$ were calculated also in bins corresponding to percentiles of $\log (L / M)$ (from $0 \%$ to $100 \%$ in steps of $10 \%$ ), by definition all populated with the same number of sources (solid line). The two lines are practically indistinguishable for $L / M<200 \mathrm{~L}_{\odot} / \mathrm{M}_{\odot}$, but the second one seems to follow a global linear behaviour over the entire range of $L / M$. A linear fit provides the empirical relation

$$
T_{\text {bol }}[K]=0.1 \times \log \left[(L / M) /\left(\mathrm{L}_{\odot} / \mathrm{M}_{\odot}\right)\right]+50.7
$$

(error bars for the two linear coefficients are $10 \%$ and $1 \%$, respectively).

Finally, a look to source surface density $\Sigma$ is given. El17 recognized an increase, on average, of this parameter from the pre-stellar to the protostellar phase. The most evolved sub-sample of this class, namely the HiI region candidates, shows a decrease of average surface density, suggesting that envelope dissipation is already ongoing at this stage. However, on the one hand this average behaviour is accompanied by a wide spread of $\Sigma$, reaching also values larger than $1 \mathrm{~g} \mathrm{~cm}^{-2}\left(A_{V}=200 \mathrm{mag}\right)$, and on the other hand no clear correlation is found between $\Sigma$ and evolutionary descriptors such as $T, L / M$, and $T_{\text {bol }}$.

Left panel of Fig. 7 suggests that, despite in this paper a more stringent filtering of HiI region candidates is made with respect to El17 in order to select a more realistic sample, the column density of sources in this sample still shows no clear evolutionary trend as a function of $L / M$. Surface density spans a wide range of about 3 orders of magnitude (central panel), and the only mild indication that can be drawn is that at very large values of the abscissa, $L / M \gtrsim 600 \mathrm{~L}_{\odot} / \mathrm{M}_{\odot}$, only surface density $\Sigma \lesssim 0.1 \mathrm{~g} \mathrm{~cm}^{-2}$ are found. Surprisingly, this is the same value of $L / M$ at which bolometric temperature is found to definitely increase over $80 \mathrm{~K}$. However, at large $L / M$ the catalogue is strongly incomplete (see Sect. 2), whereas a more robust statistics would be required to confirm a unequivocal Elia D. 
The most evolved sources in the Hi-GAL survey
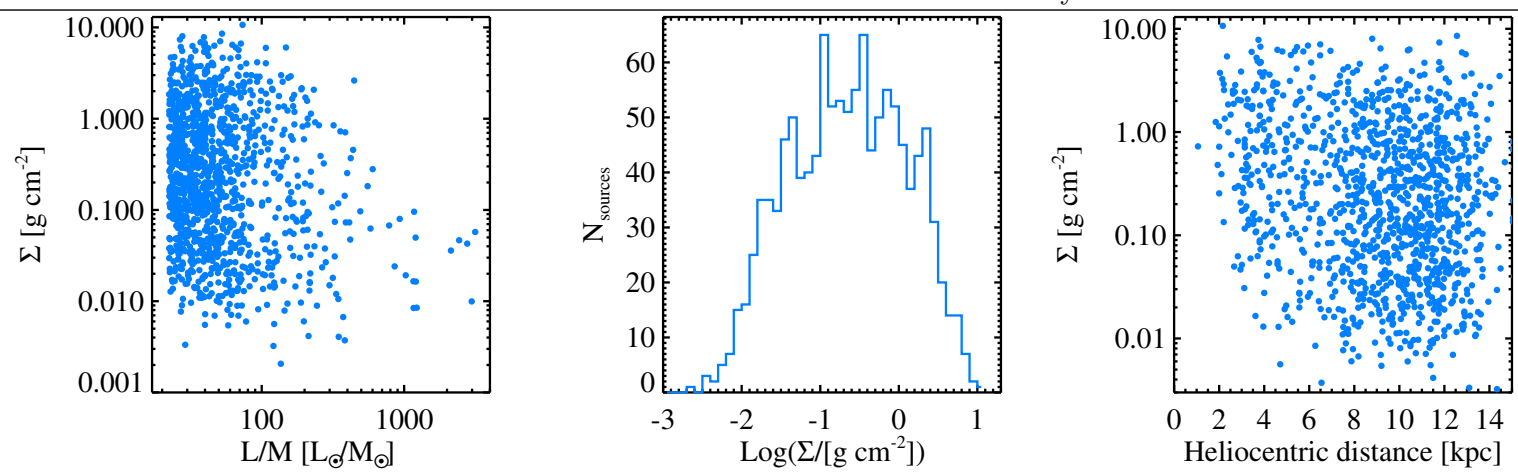

Figure 7. Diagnostics for surface density of Hi-GAL HII region candidates. Left: surface density vs ratio of bolometric luminosity to mass. Center: distribution of logarithm of column density. Right: surface density vs heliocentric distance.

trend indicating envelope dissipation at the most evolved stages probed in the Hi-GAL catalogue.

Finally, it is worth checking whether this spread of surface density may depend on a possible heliocentric distance bias. Indeed, although formally surface density is a distance-independent parameter, in practice its estimate can be affected by the distance. The Hi-GAL sources correspond to structures with different sizes and degree of internal complexity, depending on their distance. Their surface density is as an average parameter derived for the entire source, that in principle depends $i$ ) on the distribution of densities of single cores hosted by the clump and $i i$ ) on the amount of unresolved inter-core material included in such estimate. Baldeschi et al. (2017a,b) showed that global physical properties (including evolutionary indicators) of clumps detected at distances $d>>1 \mathrm{kpc}$ mirror the average properties of the contained core population; this can hinder recognition of the presence of most evolved objects in these populations. All these considerations suggest the need of a check for a possible distance bias. The surface density vs distance plot in the right panel of Fig. 7, however, again suggests no particular correlation between these two observables.

\section{Summary}

The right tail of the $L / M$ distribution of protostellar sources in the Hi-GAL catalogue, constituted by clumps candidate to host a HiI region, has been examined in detail in this work. With respect to El17, who introduced this classification, an additional constraint for source selection has been imposed, and further relations among positional, photometric, and physical properties of selected sources have been explored. The main points of this analysis can be summarised as follows:

- A sample of $1199 \mathrm{Hi}$ region candidates has been selected by imposing not only $L / M>22.4 \mathrm{~L}_{\odot} / \mathrm{M}_{\odot}$, as in El17, but also $L>1448 \mathrm{~L}_{\odot}$ as a necessary condition to have a central high-mass star.

- The distribution of Galactic positions of selected sources reveals overdensities in correspondence of spiral arm locations, but also a relevant fraction of inter-arm sources. No apparent link exists between position and evolutionary stage indicated by $L / M$, so that spiral arms do not seem to contain more evolved sources with respect to inter-arm regions.

- The dust temperature of the selected sources, estimated by fitting a modified black body to the SED at $\lambda \geq 160 \mu \mathrm{m}$, and dominated by the outer envelope of the clumps, has a median $24.4 \mathrm{~K}$, which is comparable with those found in the literature, estimated from line spectroscopy, for similar samples of sources.

- A median bolometric temperature of $52 \mathrm{~K}$ is calculated for the analysed sample, and a slightly increasing linear relation between $T_{\text {bol }}$ and $\log (L / M)$ is found $\left(T_{\text {bol }}[K]=0.1 \times \log \left[(L / M) /\left(\mathrm{L}_{\odot} / \mathrm{M}_{\odot}\right)\right]+\right.$ 50.7), once outliers of $T_{\mathrm{bol}}$ are filtered out.

- The surface density of selected sources spans about three orders of magnitude, showing no evolutionary trend. Additionally, this can not be even explained with a possible distance bias. 
The most evolved sources in the Hi-GAL survey

The source sample studied in this paper represents, at the same time, the right tail of the distribution of clump evolutionary stages than can be probed with Hi-GAL (i.e. sources still containing a relevant fraction of cold dust), and objects candidates to host high-mass stars with a surrounding HII region. To build a large statistics of bona-fide high-mass star forming objects it is necessary to carry out interferometric observations of these clumps, to resolve the level of clump-to-core fragmentation, and possibly reveal the presence of massive fragments (say $M>30 \mathrm{M}_{\odot}$ ). The ALMAGAL large ALMA Cycle 7 programme (P.I.: S. Molinari), which is being currently observed, aims at resolving the internal structure and the degree of fragmentation of 1017 far-infrared clumps, selected to be candidate to host high-mass star formation (ongoing at present or in the future) and to explore a wide variety of conditions in terms of Galactic location and source evolutionary stage. It includes 79 of the sources analysed in this paper, for which the set-up with $0.1 \mathrm{mJy}$ sensitivity will enable a complete study of the fragmentation process down to at least $1000 \mathrm{AU}$ and $0.3 \mathrm{M}_{\odot}$. This implies that possible large-mass fragments will be easily resolved, and the high-mass star forming character of these sources will be definitely ascertained.

\section{Acknowledgements}

This research is supported by INAF through the Mainstream Grant 1.05.01.86.09 "The ultimate exploitation of the Hi-GAL archive and ancillary infrared/mm data".

\section{References}

André P., Ward-Thompson D., Barsony M., 1993, ApJ, 406, 122

Baldeschi A., et al., 2017a, MNRAS, 466, 3682

Baldeschi A., Molinari S., Elia D., Pezzuto S., Schisano E., 2017b, MNRAS, 472, 1778

Cesaroni R., et al., 2015, A\&A, 579, A71

Chen H., Myers P. C., Ladd E. F., Wood D. O. S., 1995, ApJ, 445, 377

Dunham M. M., Crapsi A., Evans II N. J., Bourke T. L., Huard T. L., Myers P. C., Kauffmann J., 2008, ApJS, 179, 249

Egan M. P., et al., 2003, VizieR Online Data Catalog, 5114, 0

Elia D., et al., 2013, ApJ, 772, 45

Elia D., et al., 2017, MNRAS, 471, 100

Giannetti A., et al., 2013, A\&A, 556, A16

Giannini T., et al., 2012, A\&A, 539, A156

Gutermuth R. A., Heyer M., 2015, AJ, 149, 64

Hoare M. G., et al., 2012, PASP, 124, 939

Hofner P., Wyrowski F., Walmsley C. M., Churchwell E., 2000, ApJ, 536, 393

Hou L. G., Han J. L., Shi W. B., 2009, A\&A, 499, 473

Könyves V., et al., 2015, A\&A, 584, A91

Kroupa P., 2001, MNRAS, 322, 231

Lada C. J., 1987, in Peimbert M., Jugaku J., eds, IAU Symposium Vol. 115, Star Forming Regions. pp 1-17

Lada C. J., Wilking B. A., 1984, ApJ, 287, 610

Molinari S., et al., 2010, PASP, 122, 314

Molinari S., Merello M., Elia D., Cesaroni R., Testi L., Robitaille T., 2016, ApJL, 826, L8

Momany Y., Zaggia S., Gilmore G., Piotto G., Carraro G., Bedin L. R., de Angeli F., 2006, A\&A, 451, 515

Myers P. C., Ladd E. F., 1993, ApJL, 413, L47

Paladini R., et al., 2012, ApJ, 760, 149

Purcell C. R., et al., 2013, ApJS, 205, 1

Elia D. 
Salpeter E. E., 1955, ApJ, 121, 161

Urquhart J. S., et al., 2013, MNRAS, 435, 400

Williams S. J., Fuller G. A., Sridharan T. K., 2004, A\&A, 417, 115

Wright E. L., et al., 2010, AJ, 140, 1868 